\title{
Effect of radiotherapy fraction size on tumour control in patients with early-stage breast cancer after local tumour excision: long-term results of a randomised trial
}

\author{
J Roger Owen, Anita Ashton, Judith M Bliss, Janis Homewood, Caroline Harper, Jane Hanson, Joanne Haviland, Soren M Bentzen, \\ John R Yarnold
}

\section{Summary}

Background Standard curative schedules of radiotherapy to the breast deliver 25 fractions of $2 \cdot 0$ Gy over 5 weeks. In a randomised trial, we tested whether fewer, larger fractions were at least as safe and as effective as standard regimens. In this analysis, we assessed the long-term results of tumour control in the same population.

Methods In 1986-98, we randomly assigned 1410 women with invasive breast cancer (tumour stage 1-3 with a maximum of one positive node and no metastasis) who had had local tumour excision of early stage breast cancer to receive $50 \mathrm{~Gy}$ radiotherapy given in 25 fractions, $39 \mathrm{~Gy}$ given in 13 fractions, or 42.9 Gy given in 13 fractions, all given over 5 weeks. The primary endpoint was late change in breast appearance, which has been reported elsewhere. Here, we report ipsilateral tumour relapse, one of the secondary endpoints. Relapse was defined as any appearance of cancer in the irradiated breast. Analysis was by intention to treat.

Findings After a median follow-up of 9.7 years (IQR 7.8-11.8) for the $838(95 \%)$ patients who survived, the risk of ipsilateral tumour relapse after 10 years was $12 \cdot 1 \%(95 \%$ CI $8 \cdot 8-15 \cdot 5)$ in the 50 Gy group, $14 \cdot 8 \%(11 \cdot 2-18 \cdot 3)$ in the $39 \mathrm{~Gy}$ group, and $9.6 \%(6 \cdot 7-12 \cdot 6)$ in the 42.9 Gy group (difference between $39 \mathrm{~Gy}$ and $42 \cdot 9 \mathrm{~Gy}$ groups, $\chi^{2}$ test, $\mathrm{p}=0.027)$. The sensitivity of breast cancer to dose per fraction was estimated to be $4.0 \mathrm{~Gy}(95 \% \mathrm{CI} 1 \cdot 0-7 \cdot 8)$, similar to that estimated for the late adverse effects in healthy tissue from breast radiotherapy.

Interpretation Breast cancer tissue is probably just as sensitive to fraction size as dose-limiting healthy tissues. If this finding is confirmed, radiotherapy schedules can be greatly simplified by the delivery of fewer, larger fractions without compromising effectiveness or safety, and possibly improving both.

\section{Introduction}

The use of small fractions $(\leq 2 \cdot 0 \mathrm{~Gy})$ in curative radiotherapy is strongly based on reliable data for squamous carcinomas of the bronchus, cervix uteri, and head and neck, ${ }^{1}$ which show that these tumours are much less sensitive than late-responding healthy tissues to the size of individual fractions. If these cancers are treated with fractions of greater than $2.0 \mathrm{~Gy}$, the ratio of cured local tumours to late complications is lower than if smaller fractions are given during the same overall treatment time. ${ }^{1}$ The use of fewer fractions of more than $2.0 \mathrm{~Gy}$ (hypofractionation) in primary breast cancer is based on data suggesting that breast adenocarcinoma is more sensitive to fraction size than squamous carcinomas, and therefore could have similar fractionation sensitivity to the dose-limiting healthy tissues, including skin, subcutaneous tissues, muscle, and ribs. ${ }^{2-5}$

In classic radiobiological terms, sensitivity to fraction size is quantified by the $\alpha / \beta$ value, a variable derived from a commonly used empirical model of fractionation. The response to fraction size is typically non-linear and well fitted by a linear-quadratic function in which clinical response is proportional to $\alpha \mathrm{D}+\beta \mathrm{D}^{2}$, where $\alpha$ and $\beta$ are coefficients characteristic of the tissue under consideration and D is the fraction size. ${ }^{1}$ If breast cancer is generally as sensitive to fraction size as are the late- reacting healthy tissues of the breast, muscle, and underlying ribcage (ie, an $\alpha / \beta$ value of $3-5$ Gy compared with $\geq 10$ Gy for squamous carcinomas), larger fraction sizes will be more effective than previously thought.

A randomised clinical trial ${ }^{6}$ was therefore begun in 1986 to test the hypothesis that fewer, larger fractions are at least as effective as standard fractions of $2.0 \mathrm{~Gy}$ and offer convenience and reduced cost for patients and health services. The study was planned with late effects of healthy tissue as the primary endpoint and tumour recurrence and palpable fibrosis as the secondary endpoints. The trial was controlled for overall treatment time of radiotherapy, and generated reliable estimates of $\alpha / \beta$ of 3.6 Gy (95\% CI 1.8-5 -4) for late change in breast appearance (assessed by photographs taken before and after treatment) and of $3 \cdot 1 \mathrm{~Gy}(1 \cdot 8-4 \cdot 4)$ for moderate or striking breast induration (grade 2-3 on a clinical examination scale of 4). The early results informed the design of the UK National Cancer Research Institute (NCRI) Standardisation of Radiotherapy (START) trial, launched in January, 1999, which was planned to have sufficient statistical power to assess tumour control as the primary endpoint. A meta-analysis of the tumour control data from both trials will be done when data from the START trial mature. Here, we report on local tumour relapse in the first trial.
Lancet Oncol 2006: 7: 467-71 Published Online May 17, 2006 DOI:10.1016/S1470-2045(06) 70699-4

See Reflection and Reaction page 445

Department of Radiotherapy, Royal Marsden Hospital, Sutton, UK (J Hanson BSC Prof J R Yarnold FRCR); Department of Oncology, Gloucestershire Oncology Centre, Cheltenham, UK (J R Owen FRCR, A Ashton RCN); Clinical Trials and Statistics Unit (ICR-CTSU), Section of Clinical Trials, Institute of Cancer Research, Sutton, UK (Prof J M Bliss MSc, J Homewood BSc, C Harper MSc, J Haviland $\mathrm{MSC}$; and Department of Human Oncology, University of Wisconsin Medical School, Madison, WI, USA (Prof S M Bentzen PhD)

Correspondence to Prof John R Yarnold, Department of Radiotherapy, Royal Marsden Hospital, Downs Road, Sutton SM2 5PT, UK

john.yarnold@icr.ac.uk

$\alpha / \beta$ value

Ratio that describes sensitivity of tissues to fraction size. Low values (2-5 Gy) indicate increased tissue response for fraction sizes greater than 2.0 Gy compared with high values ( $\geq 10$ Gy) 


\section{Methods \\ Patients}

The trial was approved by the ethics committee of the Royal Marsden Hospital, Sutton, UK, in 1986. Details of the patients' characteristics have been described previously. ${ }^{6}$ Briefly, between January, 1986, and March, 1998, 1410 patients were enrolled in a clinical trial of radiotherapy dose schedules at the Royal Marsden Hospital and the Gloucestershire Oncology Centre, Cheltenham, UK. Informed consent was obtained from all patients (verbal consent was obtained early in the trial and recorded in case reports, with written consent introduced and obtained from 1991). Patients with operable invasive breast cancer (tumour [T] 1-3, node [N] $0-1$, metastasis $[\mathrm{M}] 0$ ) who needed radiotherapy were eligible for the trial, provided that they were younger than 75 years at presentation, and had had breastpreserving surgery and complete macroscopic resection of invasive carcinoma (ie, assessed by naked eye).

\section{Procedures}

We randomly allocated patients to 50 Gy given in 25 fractions of $2.0 \mathrm{~Gy}, 39$ Gy given in 13 fractions of $3.0 \mathrm{~Gy}$, or $42.9 \mathrm{~Gy}$ given in 13 fractions of $3.3 \mathrm{~Gy}$, all given over 5 weeks. We selected fraction sizes to correspond with schedules that were isoeffective (with respect to tissue response) with $50 \mathrm{~Gy}$ in 25 fractions, assuming $\alpha / \beta$ values of 1.8 Gy and $6.0 \mathrm{~Gy}$, respectively.

Randomisation was done by telephone at the Clinical Trials and Statistics Unit (ICR-CTSU) at the Institute of Cancer Research, Sutton, UK, by the clinician (early in the trial), who recorded it in the patients' notes and did not have any further role in the randomisation process, and then by a research nurse. Although randomisation was not blinded, assessments of the change in photographic breast appearance were blinded. We generated the randomisation sequence by computer. Patients were stratified by treatment centre and by the presence of microscopic foci of invasive or intraductal disease within $3 \mathrm{~mm}$ of the nearest surgical margin. Patients with a complete microscopic resection who were judged eligible by the clinician and gave consent were further randomly allocated to receive a tumourbed boost or no boost. This subrandomisation, undertaken in similar process as the main randomisation, was closed in July, 1997, and all patients were offered an elective boost thereafter. 359 patients were randomly allocated to no boost, 364 to boost, and 687 received a non-randomised boost. Patients were reviewed every 3 months for the first 3 years, every 6 months for the next 5 years, and every year thereafter. The radiotherapy technique has been reported elsewhere. ${ }^{6}$ Patients were simulated (ie, imaged before

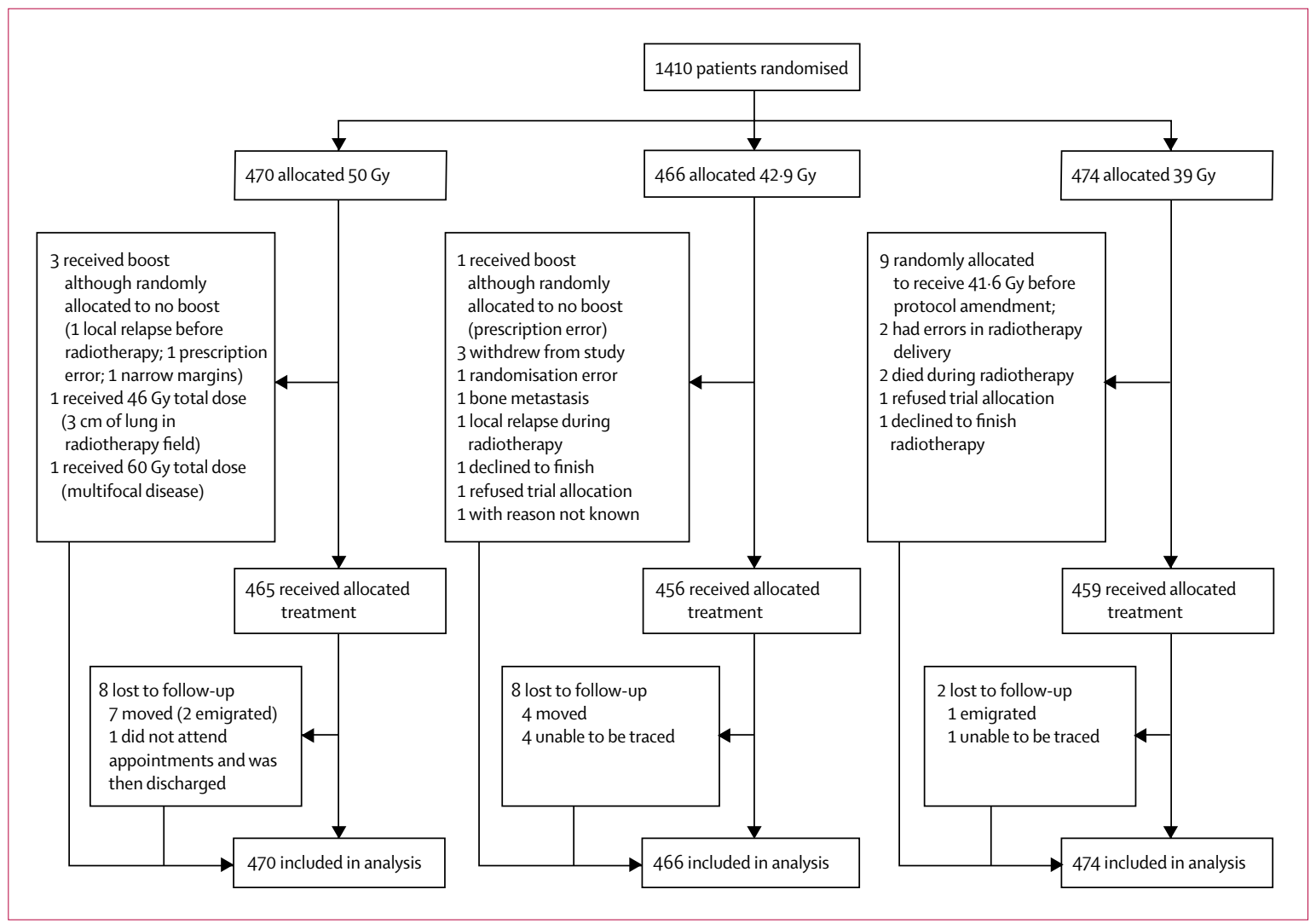

Figure 1: Trial profile 


\begin{tabular}{|c|c|c|c|c|c|c|}
\hline & \multirow[t]{2}{*}{$\begin{array}{l}\text { Number of local relapses/ } \\
\text { person-years }\end{array}$} & \multirow[t]{2}{*}{$\begin{array}{l}\text { Crude hazard ratio } \\
(95 \% \mathrm{Cl})^{\star}\end{array}$} & \multicolumn{2}{|c|}{$\begin{array}{l}\text { Kaplan-Meier estimate of local recurrence } \\
(95 \% \mathrm{Cl})\end{array}$} & \multicolumn{2}{|c|}{$\begin{array}{l}\text { Smoothed estimate of absolute difference in local recurrence } \\
(95 \% \mathrm{Cl})^{*}\end{array}$} \\
\hline & & & 5 years' follow-up & 10 years' follow-up & 5 years' follow-up & 10 years' follow-up \\
\hline $50 \mathrm{~Gy}$ & $50 / 3965$ & 1 & $7 \cdot 9 \%(5 \cdot 4-10 \cdot 4)$ & $12 \cdot 1 \%(8 \cdot 8-15 \cdot 5)$ & .. & .. \\
\hline $42.9 \mathrm{~Gy}$ & $42 / 3840$ & $0.86(0.57-1.30)$ & $7 \cdot 1 \%(4 \cdot 6-9 \cdot 5)$ & $9 \cdot 6 \%(6 \cdot 7-12 \cdot 6)$ & $-1 \cdot 1 \%(-3 \cdot 3-2 \cdot 3)$ & $-1 \cdot 6 \%(-5 \cdot 0-3 \cdot 3)$ \\
\hline $39 \mathrm{~Gy}$ & $66 / 3890$ & $1.33(0.92-1.92)$ & $9 \cdot 1 \%(6 \cdot 4-11 \cdot 7)$ & $14 \cdot 8 \%(11 \cdot 2-18 \cdot 3)$ & $2 \cdot 5 \%(-0.6-6 \cdot 7)$ & $3.7 \%(-0 \cdot 9-9 \cdot 8)$ \\
\hline
\end{tabular}

treatment for planning purposes) and treated in the same supine position. 6-MV x-rays were used for most patients, although some smaller-sized patients $(n=333)$ received Cobalt-60 $\gamma$-rays or 4 MV x-rays and 10 MV x-rays for larger-sized patients $(n=2)$. To classify patients by size, an arbitrary scale (small, medium, or large) was used by three independent observers to the baseline photographs of the breast. Intra-observer and inter-observer measures of agreement showed this system for size to be a good enough descriptor for our purposes.

We used wedge tissue compensators in all patients, with the wedge angles estimated from one transverse external contour through the central plane. The reference point for tangential fields was in the centre of the breast, midway between the skin entry points of the tangential fields, and midway between a perpendicular line from the skin surface to the interface of the lung and chest wall. Once delivered, the lymph nodes were treated with an anterior field to the supraclavicular fossa, prescribed as an applied dose. If the axilla was included, a posterior axillary field of equal weight was treated with every fraction, to ensure that $100 \%$ of the prescribed dose was delivered to the axillary midline.

Boosts were delivered by electrons to the tumour bed with a dose of $14 \mathrm{~Gy}$ (given as seven 2-Gy fractions per day) to the $90 \%$ isodose ( $15 \cdot 5$ Gy to $100 \%)$. The proportion of patients who received a boost was almost identical in all three treatment groups: 348 (74\%) patients for $50 \mathrm{~Gy}$, 348 (75\%) for $42.9 \mathrm{~Gy}$, and 351 (74\%) for $39 \mathrm{~Gy}$.

As the endpoint for this analysis, local relapse was defined as any malignant disease that developed in the ipsilateral breast parenchyma or overlying skin (recurrence is defined as regrowth of original tumour). Distant relapse was defined as any appearance of malignant disease outside the ipsilateral breast and regional lymph nodes (ipsilateral axilla or supraclavicular fossa). Contralateral cancer was defined as any appearance of malignant tumour in the contralateral breast. Local relapse and contralateral primary tumours were always confirmed histologically, whereas metastases could be used as a clinical diagnosis in the event of any uncertainty (biopsy of internal organs can be hazardous or difficult to undertake).

\section{Statistical analysis}

The original sample size $(n=1410)$ was estimated on the basis of treatment not increasing the occurrence of moderate or severe late radiation effects in healthy tissues to more than $10 \%$ (in the assumption of $<5 \%$ in the 50 Gy group), which was the primary endpoint of the trial and has been reported elsewhere. ${ }^{6}$ Midway through the trial, we decided to extend the trial to recruit more patients and to allow a reliable comparison of tumour recurrence between the dose groups, which we report here. For an estimated $90 \%$ power and $5 \%$ significance level, 2250 patients would be needed to detect a $5 \%$ absolute increase in the risk of recurrence in either experimental group (42.9 Gy and $39 \mathrm{~Gy}$ ), compared with an expected 5-year local recurrence of $10 \%$ in the control group ( $50 \mathrm{~Gy}$ ). Accrual was stopped before the target was reached, because this trial was superseded by the START trial, a national initiative that investigated fraction sizes of more than $2 \cdot 0 \mathrm{~Gy}$ in breast cancer, with tumour control as the primary endpoint.

We calculated the time from randomisation to first recurrence, date last seen, or date of death, whichever occurred first. We used Kaplan-Meier to calculate the probability of no local recurrence at 5 and 10 years' follow-up, and fractionation schedules were compared by use of the log-rank test. Cox proportional hazards regression was used to estimate the hazard ratios (with $95 \% \mathrm{CI}$ ) for local recurrence in every fractionation schedule. Since point estimates of differences in events can, by chance, be atypical of the overall pattern of differences, we obtained smoothed estimates of absolute differences in recurrence at 5 and 10 years by using the hazard ratios obtained from the Cox model and the Kaplan-Meier estimate of local recurrence in the control group. ${ }^{8}$ We obtained a direct estimate of the $\alpha / \beta$ ratio for local recurrence by fitting a Cox proportional hazards regression model containing a term for total dose, and another representing total dose multiplied by dose per fraction. The $\alpha / \beta$ ratio was then calculated by dividing the two parameter estimates (estimate for total dose $\div$ [estimate for total dosexdose per fraction]). Analysis was on an intention-to-treat basis.

\section{Role of the funding source}

The sponsors of the study had no role in study design, data collection, data analysis, data interpretation, or writing of the report. The corresponding author had full access to all the data in the study and had final responsibility for the decision to submit for publication. 


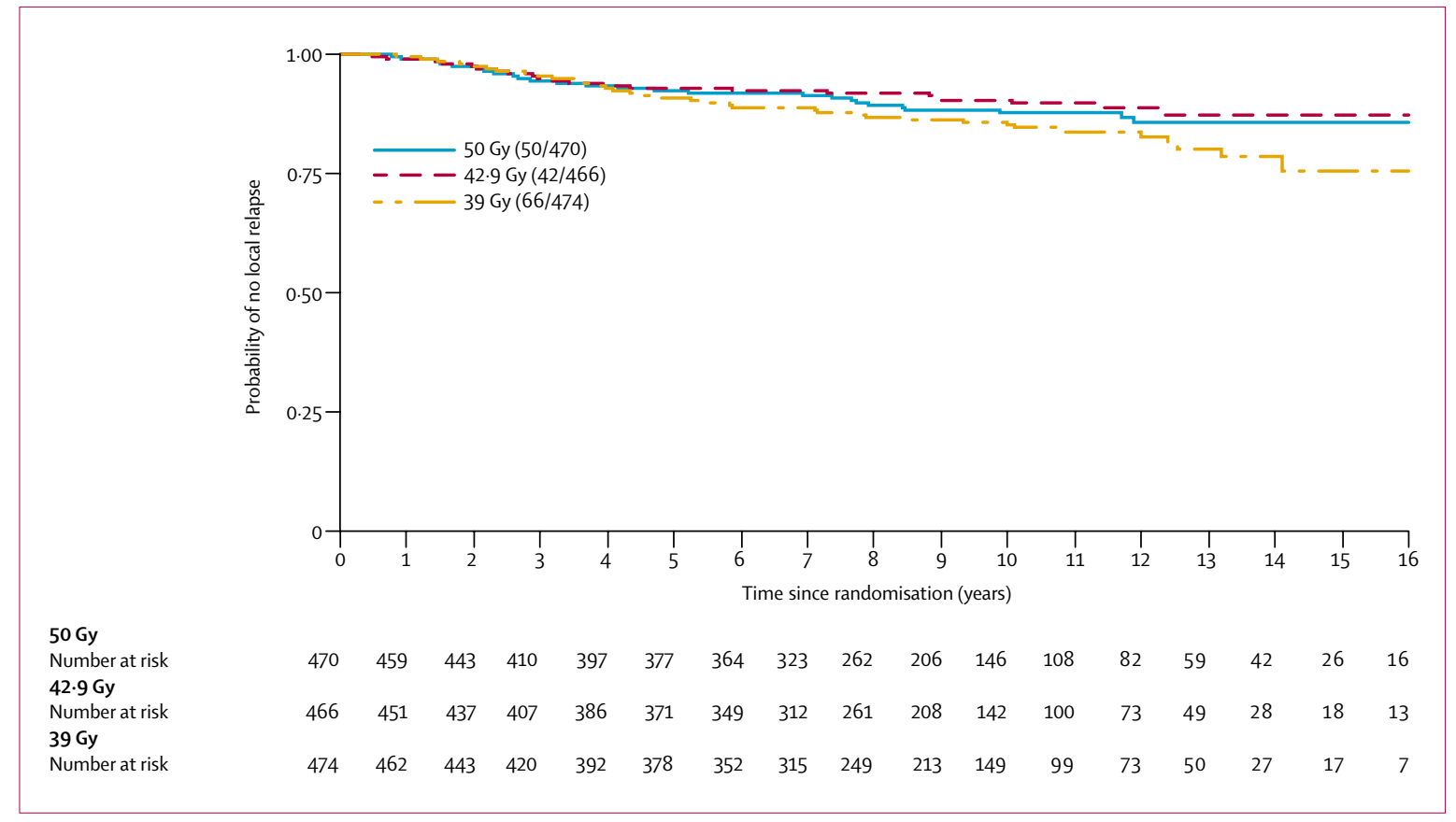

Figure 2: Local ipsilateral relapse in the breast according to fractionation schedule

\section{Results}

1410 patients were recruited in the trial (figure 1). Median follow-up of patients still alive was $9 \cdot 7$ years (IQR 7.8-11.8), with a maximum follow-up of 18.4 years. $18(1 \%)$ patients were lost to follow-up (median follow-up $4 \cdot 9$ years, IQR 2.5-8·3). At the time of analysis, 838 patients $(59 \%)$ were alive and without local relapse, 46 (3\%) were alive with local recurrence (but with no distant relapse or contralateral breast cancer), 46 (3\%) were alive with distant relapse (including ten with local relapse and five with contralateral breast cancer), 35 (2\%) were alive with a second primary cancer in the contralateral breast (including three with local relapse), and 445 (32\%) had died (of whom 99 had a local relapse).

At the time of the analysis, 158 (11\%) patients had had local relapse. Local recurrence was highest between years 3 and 5 of follow-up, and 106 (67\%) events took place within 5 years of follow-up. The table and figure 2 show the survival analysis of local recurrence, comparing the three fractionation schedules. The probability of local recurrence between the $42 \cdot 9$ Gy and 39 Gy groups differed significantly (smoothed estimate of absolute difference $3 \cdot 7 \%, 95 \%$ CI $0 \cdot 3-8 \cdot 3 ; \chi^{2}$ test, degrees of freedom $[\mathrm{df}]=1, \mathrm{p}=0 \cdot 027$ ).

Figure 2 shows that recurrence-free survival curves for the fractionation schedules diverge only after 5 years of follow-up. However, Cox models for proportional hazard regression showed hazard ratios of $0.90(95 \% \mathrm{CI}$ 0.55-1.46) for 42.9 Gy and 1.14 (0.72-1.79) for $39 \mathrm{~Gy}$, compared with $50 \mathrm{~Gy}$ in the first 5 years' follow-up, and $0.77(0.36-1.69)$ for 42.9 Gy and $1.81(0.96-3.41)$ for
$39 \mathrm{~Gy}$ in the follow-up from 5 years onwards. However, the test of the proportional hazards assumption was not significant $(\mathrm{p}=0 \cdot 1)$, indicating that the apparent differences between the two periods were not substantial.

From the Cox proportional hazard regression, the direct estimate of the $\alpha / \beta$ ratios for local recurrence was $4 \cdot 0 \mathrm{~Gy}(95 \% \mathrm{CI} 1 \cdot 0-7 \cdot 8)$. By using this value to estimate the equivalent total doses in fractions of $2.0 \mathrm{~Gy}$ (with respect to tissue response; malignant as well as benign tissue), we calculated that $39 \mathrm{~Gy}$ in 13 fractions of $3.0 \mathrm{~Gy}$ would be equivalent (have the same $\alpha / \beta$ value) to $46 \mathrm{~Gy}$ in 23 fractions of $2.0 \mathrm{~Gy}$, and that $42.9 \mathrm{~Gy}$ in 13 fractions of 3.3 Gy over 5 weeks would be isoeffective with $52 \mathrm{~Gy}$ in 26 fractions of $2.0 \mathrm{~Gy}$. By interpolation, $50 \mathrm{~Gy}$ in 25 fractions of 2.0 Gy would be isoeffective with $41.6 \mathrm{~Gy}$ in 13 fractions of $3 \cdot 2$ Gy for 5 weeks. These isoeffective doses allow an estimation of the local steepness of the dose-response curve, the normalised dose-response gradient ( $\gamma$ value), corresponding to a $0.5 \%$ increase in local relapse probability for a $1 \%$ increase in total dose. Thus, a $2 \%$ absolute improvement in local control is the result of an increment of $2 \mathrm{~Gy}$ in total dose.

\section{Discussion}

Based on an analysis of 158 events of ipsilateral local tumour relapse, we have shown that the estimated sensitivity of breast cancer to fraction size (ie, $\alpha / \beta$ value) of $4.0 \mathrm{~Gy}$ is closely similar to that of $3.6 \mathrm{~Gy}$ for any change in breast appearance, ${ }^{6}$ and much the same as that of $3.1 \mathrm{~Gy}$ for moderate to substantial induration. ${ }^{6}$ An important finding from this study is the significant 
relation between dose response and local tumour relapse. A $\gamma$ value of 0.5 might seem low, but in adjuvant treatment in general the dose-response curve for tumour relapse does not range from $0 \%$ to $100 \%$, since about $70 \%$ of the patients achieve local control by surgery alone. ${ }^{9}$ The outcome of the present trial is consistent with modelling based on the results of the EORTC radiotherapy breast boost trial. ${ }^{10}$

With the assumption of linearity between the two test doses, a schedule of $41 \cdot 6$ Gy in 13 fractions of $3 \cdot 2 \mathrm{~Gy}$ for 5 weeks is equivalent to $50 \mathrm{~Gy}$ in 25 fractions of $2.0 \mathrm{~Gy}$ with respect to tumour recurrence, although the 95\% CIs remain wide. For reliable estimates of the fractionation sensitivity of breast cancer, results of the UK START Trial A of 2236 patients (International Standard Randomised Controlled Trial Number 59368779) are awaited, which includes randomised comparisons of $41.6 \mathrm{~Gy}$ in 13 fractions of $3 \cdot 2 \mathrm{~Gy}$ and 39.0 Gy in 13 fractions of 3.0 Gy over 5 weeks with a control schedule of $50 \mathrm{~Gy}$ in 25 fractions of $2 \cdot 0 \mathrm{~Gy}$. Results from a Canadian randomised trial of 1234 patients showed no difference in ipsilateral tumour relapse, based on 44 tumour events, following $50 \mathrm{~Gy}$ in 25 fractions of $2 \cdot 0$ Gy over 35 days and $42 \cdot 5$ Gy in 16 fractions of 2.66 Gy over 22 days to the whole breast after excision of microscopic tumour. ${ }^{11}$ A comparison based on 44 events is very imprecise, but if the 25-fraction and 16-fraction schedules are truly isoeffective for tumour control, and if no difference in relapse between schedules is assumed, the $\alpha / \beta$ value for tumour response could be as low $3.0 \mathrm{~Gy}$, which is consistent with the fractionation sensitivity of the doselimiting responses of healthy tissue that develop years later. Meanwhile, the results of the UK START Trial B, which randomly assigned 2215 women to $40 \mathrm{~Gy}$ in 15 fractions over 3 weeks or to 50 Gy in 25 fractions over 5 weeks, will strengthen the interpretation of the Canadian trial.

The clinical implications for patients with breast cancer and health services will be profound if the fractionation sensitivity of breast cancer is shown to be similar to that of the dose-limiting healthy tissues of the breast, including tissue from the underlying pectoral muscle and ribcage. Although the data do not apply to treatment of the lymphatic pathways, where the brachial plexus is known to be highly sensitive to fraction size, there would be no reason to prefer $2 \cdot 0 \mathrm{~Gy}$ fractions for most women who need radiotherapy to the conserved breast or postmastectomy chest wall. The challenge will be to determine the useful limits of hypofractionation. Current initiatives include the randomised UK FAST Trial, ${ }^{12}$ which compares two doses $(5 \cdot 7 \mathrm{~Gy}$ and $6 \cdot 0 \mathrm{~Gy})$ in five fractions over 5 weeks with a control dose of
$50 \mathrm{~Gy}$ in 25 fractions, delivered with three-dimensional, dose-compensated whole-breast radiotherapy. However, although our trial results are consistent with the hypothesis, they are not sufficiently reliable on their own to justify hypofractionation outside the context of well-designed randomised trials.

Conflicts of interest

We declare no conflicts of interest.

\section{Contributors}

J R Owen, J Bliss, and J R Yarnold participated in the design, conduct, and analysis of the outcome of this trial, and contributed to the writing of the manuscript. A Ashton, J Homewood, C Harper, and J Hanson participated in the conduct and analyses of the outcome of this trial. J Haviland and S M Bentzen participated in the analysis of the outcome of this trial and contributed to the writing of the manuscript. All the authors saw and approved the final version.

\section{Acknowledgments}

We thank all the patients who participated in this study. We also thank many colleagues who contributed to the design, implementation, and analysis of this study for many years, including: Ingela Turesson, University Hospital, Uppsala, Sweden; Mike Henk and Diana Tait, Royal Marsden Hospital, Sutton, UK; Stephen Ebbs, Mayday University Hospital, Croydon, UK; Kim Benstead and Sean Elyan, Gloucestershire Oncology Centre, Cheltenham, UK. The trial was partly supported in its early years by a grant from Marks and Spencer PLC. The ICR-CTSU receives funding from Cancer Research UK.

\section{References}

1 Bentzen SM, Baumann M. The linear-quadratic model in clinical practice. In: Steel GG, ed. Basic clinical radiobiology, 3rd edn. London: Arnold, 2002: 134-46.

2 Thames HD, Hendry JH, Moore JV, et al. The high steepness of dose-response curves for late-responding normal tissues. Radiother Oncol 1989; 15: 49-53.

3 Thames HD, Bentzen SM, Turesson I, et al. Time-dose factors in radiotherapy: a review of the human data. Radiother Oncol 1990; 19: 219-35.

4 Cohen L. Radiotherapy in breast cancer I. The dose-time relationship theoretical considerations. Br J Radiol 1952; 25: 636-42.

5 Douglas BG, Castro JR. Novel fractionation schemes and high linear energy transfer. Prog Exp Tumor Res 1984; 28: 152-65.

6 Yarnold J, Ashton A, Bliss J, et al. Fractionation sensitivity and dose response of late adverse effects in the breast after radiotherapy for early breast cancer: long-term results of a randomised trial. Radiother Oncol 2005; 75: 9-17.

7 START trial management group. Standardisation of Breast Radiotherapy (START) Trial. Clin Oncol 1999; 11: 145-47.

8 Machin D, Cheung YB, Parmar MKB. Survival analysis: a practical approach, 2nd edn. Chichester, UK: John Wiley and Sons Ltd, 2006.

9 Bentzen SM. High-tech in radiation oncology: should there be a ceiling? Int J Radiat Oncol Biol Phys 2004; 58: 320-30.

10 Bartelink H, Horiot JC, Poortmans P, et al. Recurrence rates after treatment of breast cancer with standard radiotherapy with or without additional radiation. N Engl J Med 2001; 345: 1378-87.

11 Whelan T, MacKenzie R, Julian J, et al. Randomized trial of breast irradiation schedules after lumpectomy for women with lymph node-negative breast cancer. J Natl Cancer Inst 2002; 94: 1143-50.

12 Yarnold J, Bloomfield D, LeVay J, et al. Prospective randomised trial testing 5.7Gy and 6.0Gy fractions of whole breast radiotherapy in women with early breast cancer (FAST) trial. Clin Oncol 2004; 16: S30. 